\title{
Utility of Contrast-Enhanced Magnetic Resonance Angiography for Delayed Intracranial In-Stent Stenosis in Nonatherosclerotic Cerebral Vascular Diseases
}

\author{
Santhosh Kumar Kannath ${ }^{1}$ Jayadevan Enakshy Rajan ${ }^{1}$ Kamble Jayaprakash Harsha ${ }^{2}$ \\ ${ }^{1}$ Department of Imaging Sciences and Interventional Radiology, \\ Neurointervention Center, Sree Chitra Tirunal Institute of Medical \\ Sciences and Technology, Trivandrum, Kerala, India \\ 2 Department of Imaging Sciences and Interventional Radiology, \\ Sree Chitra Institute for Medical Sciences and Technology, \\ Thiruvananthpuram, Kerala, India \\ Address for correspondence Jayadevan Enakshy Rajan, DM, \\ Department of Imaging Sciences and Interventional Radiology, \\ Neurointervention Center, Sree Chitra Tirunal Institute of Medical \\ Sciences and Technology, Trivandrum, Kerala 695011, India \\ (e-mail: drjayadevan@gmail.com).
}

J Clin Interv Radiol ISVIR 2017;1:85-88.

\begin{abstract}
Keywords

- intracranial stent

- in-stent stenosis

- magnetic resonance angiography

- MRA

- digital subtraction angiography

Noninvasive imaging modalities are being used for long-term follow-up of intracranial stented patients of nonatherosclerotic etiology. The aim of this study is to determine the utility of contrast-enhanced magnetic resonance angiography (CE-MRA) source images in delayed intracranial in-stent stenosis. A total of 18 stented patients for nonatherosclerotic etiology were reviewed; all had follow-up digital subtraction angiography (DSA) and CE- and time-of-flight (TOF)-MRA. Four sets of MR images (TOF-MRA reformatted images, TOF-MRA source images, CE-MRA reformatted images, and CE-MRA source images) were reviewed for detection of $\geq 50 \%$ stenosis. Accuracy of each image set was calculated comparing to DSA. Overall delayed in-stent stenosis during follow-up DSA was $10 \%$. The sensitivity of TOF reformatted image, TOF source image, CE-MRA reformatted image, CE-MRA source image are $33 \%(6 / 18), 55.6 \%(10 / 18), 77.8 \%(14 / 18)$, and $100 \%(18 / 18)$, respectively, while negative predictive value are $14.3 \%(2 / 14), 20 \%(2 / 10), 33 \%(2 / 6)$, and $100 \%(2 / 2)$, respectively. CE-MRA source images are equally efficacious as DSA to detect significant $(\geq 50 \%$ ) delayed in-stent stenosis.
\end{abstract}

\section{Introduction}

In-stent stenosis is a well-described delayed complication of angioplasty and stenting for atheromatous disease. ${ }^{1,2}$ Detection of high-risk in-stent restenosis would identify a subgroup of patients who may benefit from close observation/ early treatment. Digital subtraction angiography (DSA) is the gold standard imaging modality for follow-up of stent patency, but is invasive in nature and associated complication rate (range: $1.8-2.1 \%$ ) would limit its use for repeated and long-term evaluation. Various noninvasive imaging modalities are described for in-stent stenosis evaluation. However, exact accuracy of each modality is not widely studied.

Based on previous evidence of SSYLVIA trial, ${ }^{1}$ in which stenosis $>50 \%$ was grouped as high-risk group, the delayed in-stent stenosis in our study also defined $>50 \%$ delayed instent stenosis as "significant stenosis" requiring at least clinical attention/close follow-up/intervention. Hence, we aimed to study accuracy of contrast-enhanced magnetic resonance angiography (CE-MRA) and time-of-flight (TOF)-MRA in detection of significant delayed in-stent stenosis as compared with DSA.

\section{Material and Methods}

\section{Patient Selection}

The study was a retrospective-prospective evaluation of patients who underwent endovascular stent placement in intracranial circulation (defined as cavernous internal carotid artery and beyond, $\mathrm{V} 4$ vertebral artery and beyond), who had follow-up MRA and DSA during January 2009 to 
December 2015. Patients treated with covered stent and stented for atherosclerotic stenosis were excluded from the study. In addition, interval of more than 1 month between MRA and DSA were excluded. Medical records were reviewed for patient demographic details such as age, sex, clinical presentation, DSA and MRA study, treatment given including medical and endovascular therapy, and follow-up. A few patients' MRA was retrospectively evaluated, while a few patients were prospectively followed with DSA/MRA.

\section{Image Acquisition}

MR imaging of the brain was performed using a 12-channel matrix coil on a $1.5 \mathrm{~T}$ clinical scanner (Avanto, SQ Engine; Siemens, Erlangen, Germany). Digital subtraction angiogram was obtained using a biplane neuro angiographic suit (Innova 3131, GE Medical systems, LLC, Milwaukee, Wisconsin, United States) with standard protocols, by transfemoral route and selective acquisition of vessels of interest in standard angiographic projections by iodinated contrast injection (Omnipaque 320; GE Healthcare, Princeton, New Jersey, United States).

\section{Image Analysis}

Four sets of MR images (TOF-MRA volume-rendered images, TOF-MRA source images, CE-MRA volume-rendered images, CE-MRA source images) were evaluated independently and in a randomized fashion by two experienced neuroradiologists (experience of reader 1: 10 years and reader 2: 5.5 years) and any deviation was resolved by consensus. Percentage stenosis was measured by using following formula, percent stenosis $=(1-$ smallest in-stent diameter/diameter at the proximal $/$ distal normal vessel segment $) \times 100$. This calculation is a onedimensional view. If there are poor quality images in any one of the sets, the entire patient image sets were excluded from the study. We defined "significant in-stent stenosis" as $\geq 50 \%$ stenosis requiring clinical attention and further evaluation/ intervention. "Delayed" in-stent stenosis was defined as instent stenosis 3 months after stent deployment. The stenosis in five sets of images (1 DSA + 4 MRA) was grouped into $<50 \%$ and $\geq 50 \%$ stenosis for statistical analysis.

\section{Statistical Analysis}

A commercially available statistical software package (Statistical Package for the Social Sciences, Version 17; SPSS, Chicago, Illinois, United States) was used for analysis. Fisher's exact test was used for statistical significance evaluation. The $p$ value of $<0.05$ was regarded as a statistically significant result. Sensitivity, specificity, accuracy, and positive and negative predictive values of different imaging sets against DSA were calculated. Statistical significance of CE-MRA source images was also calculated between Leo stent (Balt, Montmorency, France) and Neuroform stent (Stryker, Kalamazoo, Michigan, United States).

\section{Results}

Overall, 38 patients with intracranial stent deployment for various etiologies were identified. A total of 18 stented patients for nonatherosclerotic etiology had follow-up DSA; all the 18 patients had at least one follow-up MRA, 6 patients had two follow-up MRA, 1 each had three and four follow-up MRAs. - Table 1 summarizes the demographics of stented patients, - Fig. 1 describes in-stent stenosis detection in different imaging modalities as compared with DSA.

Overall delayed in-stent stenosis during follow-up DSA was $11 \%$, which is slightly high as compared with $7.8 \%$ of previously published literature. ${ }^{3}$ CE-MRA source image are

Table 1 Demographics of stented patients

\begin{tabular}{|c|c|}
\hline Age distribution $(N=18)$ & $\begin{array}{l}\text { Mean age was } 41.5 \text { y } \\
\text { (range } 12-80 \mathrm{y} \text { ) }\end{array}$ \\
\hline $\begin{array}{l}\text { Sex distribution of } \\
\text { patients }(N=18)\end{array}$ & $\mathrm{M}: \mathrm{F}=1: 1$ \\
\hline Duration of follow-up $(N=18)$ & $\begin{array}{l}\text { Mean follow-up } 17.5 \text { mo } \\
\text { (range 4-62 mo) }\end{array}$ \\
\hline \multicolumn{2}{|l|}{ Etiology for stenting $(N=18)$} \\
\hline Stent-assisted coiling & $16(88.9 \%)$ \\
\hline Direct CCF & $1(5.5 \%)$ \\
\hline Nonatherosclerotic stenosis & $1(5.5 \%)$ \\
\hline \multicolumn{2}{|l|}{ Location of stents $(N=18)$} \\
\hline Anterior circulation & $11(60 \%)$ \\
\hline Posterior circulation & $7(40 \%)$ \\
\hline \multicolumn{2}{|c|}{ Distribution of stents in various intracranial vessels } \\
\hline Cavernous ICA & $3(16 \%)$ \\
\hline $\begin{array}{l}\text { Communicating } \\
\text { segment ICA }\end{array}$ & $6(33 \%)$ \\
\hline MCA & $2(11 \%)$ \\
\hline $\mathrm{BA}$ & $3(16 \%)$ \\
\hline BA-PCA & $2(11 \%)$ \\
\hline VA-basilar artery & $1(6 \%)$ \\
\hline \multicolumn{2}{|l|}{ Type of stents used $(N=18)$} \\
\hline Neuroform 3 & $8(44 \%)$ \\
\hline Leo & $6(33 \%)$ \\
\hline Solitaire AB & $3(17 \%)$ \\
\hline Enterprise & $1(6 \%)$ \\
\hline \multicolumn{2}{|l|}{ Delayed in-stent stenosis in DSA } \\
\hline Neuroform 3 & 0 \\
\hline Leo & 0 \\
\hline Solitaire $A B$ & 1 \\
\hline Enterprise & 0 \\
\hline \multicolumn{2}{|l|}{ Delayed stent occlusion in DSA } \\
\hline Neuroform 3 & 0 \\
\hline Leo & 1 \\
\hline Solitaire AB & 0 \\
\hline Enterprise & 0 \\
\hline
\end{tabular}

Abbreviations: BA, basilar artery; CCF, carotid cavernous fistula; DSA, digital subtraction angiography; ICA, internal carotid artery; MCA, carotid cavernous fistula; PCA, posterior cerebral artery; VA, vertebral artery. 

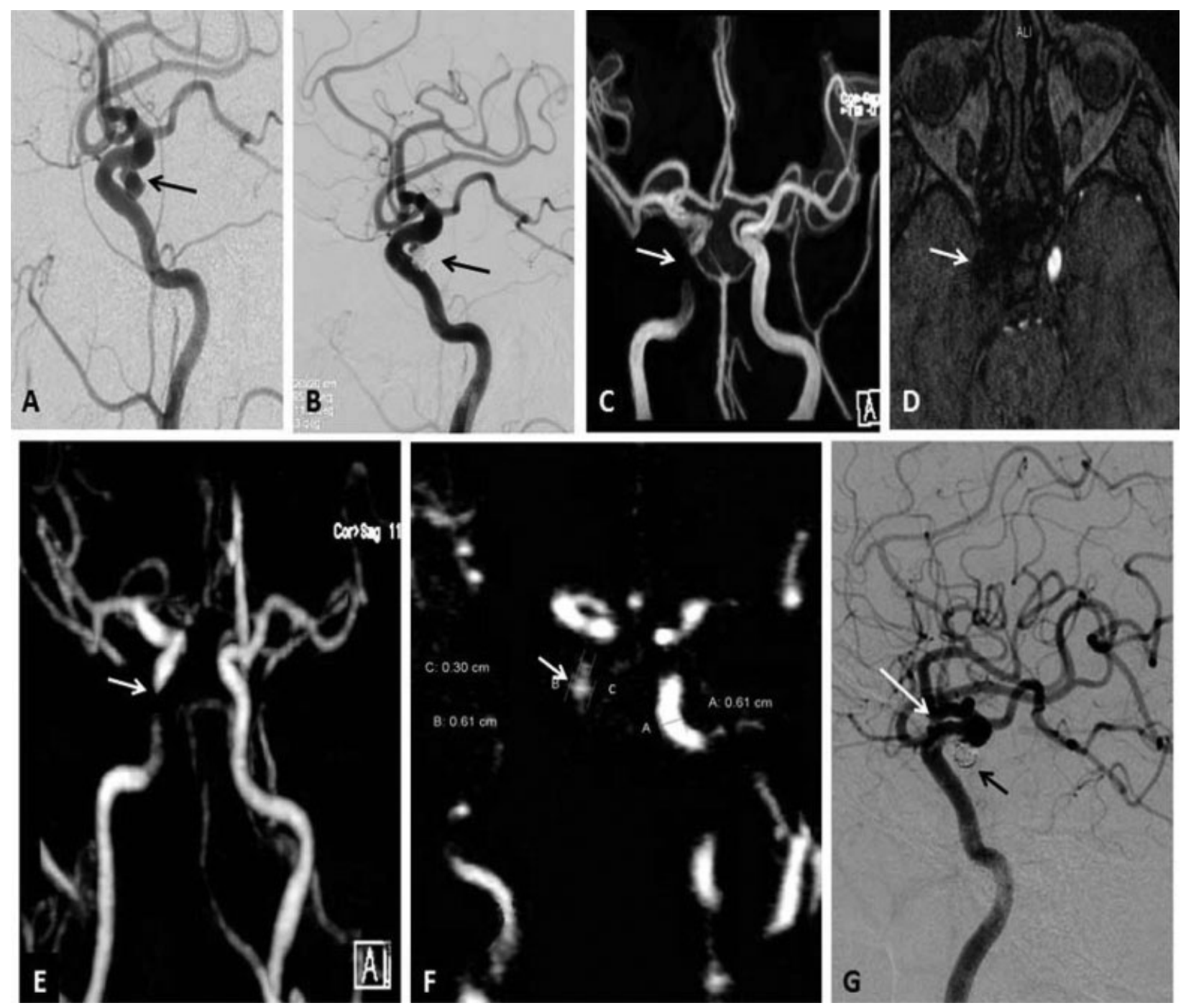

Fig. 1 Comparison of various MRA images with DSA. Preprocedure DSA-saccular aneurysm in the communicating segment of ICA (A), Immediate postprocedure DSA after stent-assisted coiling (B), follow-up TOF-MRA reformatted image-interruption of ICA at the level of stent (C), TOF-MRA source image-no definite evidence of flow in the ICA (D), CE-MRA reformatted image-interruption of ICA at the stented segment (E), CE-MRA source image-more than $50 \%$ stenosis of ICA (F), postprocedure DSA-stenosis of ICA at the proximal part of stent (G). CE, contrastenhanced; DSA, digital subtraction angiography; ICA, internal carotid artery; MRA, magnetic resonance angiography; TOF, time-of-flight.

$100 \%$ sensitivity, specificity, positive predictive value, and negative predictive value in detecting significant delayed instent stenosis ( $\geq 50 \%$ stenosis). However, specificity and positive predictive value of all four MRA image sets was $100 \%$, the sensitivity and negative predictive value was significantly different in four image sets. The sensitivity of
TOF reformatted image, TOF source image, CE-MRA reformatted image, CE-MRA source image are 33\% (6/18), 55.6\% (10/18), $77.8 \%$ (14/18), and $100 \%$ (18/18), respectively, while negative predictive value are $14.3 \%$ (2/14), $20 \%(2 / 10), 33 \%$ $(2 / 6)$, and $100 \%(2 / 2)$, respectively (-Table 2). Patient compliance of antiplatelet therapy was $100 \%$ in all 18 cases,

Table 2 Comparison of sensitivity, specificity, PPV, NPV compared against DSA for detection of significant ( $\geq 50 \%$ ) delayed in-stent stenosis

\begin{tabular}{|l|l|l|l|l|l|}
\hline & $\boldsymbol{p}$ Value & Sensitivity & Specificity & PPV & NPV \\
\hline CE MRA SI & 1.000 & $100 \%(18 / 18)$ & $100 \%(2 / 2)$ & $100 \%(18 / 18)$ & $100 \%(2 / 2)$ \\
\hline CE reformatted image & 0.125 & $77.8 \%(14 / 18)$ & $100 \%(2 / 2)$ & $100 \%(14 / 14)$ & $33 \%(2 / 6)$ \\
\hline TOF MRA SI & 0.008 & $55.6 \%(10 / 18)$ & $100 \%(2 / 2)$ & $100 \%(10 / 10)$ & $20 \%(2 / 10)$ \\
\hline TOF reformatted image & 0.000 & $33 \%(6 / 18)$ & $100 \%(2 / 2)$ & $100 \%(6 / 6)$ & $14.3 \%(2 / 14)$ \\
\hline
\end{tabular}

Abbreviations: CE, contrast-enhanced; DSA, digital subtraction angiography; MRA, magnetic resonance angiography; NPV, negative predictive value; PPV, positive predictive value; SI, source image; TOF, time-of-flight. 
indicating the lesser role of platelet activation by stent as a direct cause for delayed in-stent stenosis.

CE-MRA reformatted image, though less sensitive as compared with CE-MRA source image, both these image sets showed no statistically significant difference. In addition, the reformatted images were also statistically insignificant as compared with DSA in detection of $>50 \%$ stenosis.

\section{Discussion}

Delayed in-stent stenosis is increasingly identified in different stents, though exact treatment remains controversial. The reported incidence of Neuroform in-stent stenosis is $5.8 \%$ in patients with symptomatic intracranial stenosis. ${ }^{4}$ Intracranially stented patients need frequent and longterm follow-up for stent patency evaluation. Hence, different noninvasive modalities are being evaluated for delayed instent stenosis. Modification of regular computed tomography angiogram and contrast enhanced trans cranial Doppler (CE-TCD) is found to be useful for intracranial stenosis evaluation, ${ }^{5,6}$ a case report of utility of CE-TCD in intracranial stent evaluation also been described. ${ }^{7}$

Though the utility of TOF-MRA and CE-MRA in in-stent stenosis has been described, accuracy of each modality was not been evaluated in detail. In a recent study of various MRA techniques for the evaluation of in-stent flow, CE-MRA showed better flow signals. ${ }^{8}$ In our study, CE-MRA identified significant stenosis ( $\geq 50 \%$ stenosis + occlusion) correctly in all cases. However, as compared with DSA, CE-MRA showed 7 (35\%) cases of less than $50 \%$ stenosis which is significantly less than previously reported $64 \%$.

One of asymptomatic patient revealed 50\% stenosis of Neuroform stent during follow-up angiography; he was treated with tirofiban infusion. Following tirofiban infusion, the stenosis reduced significantly. The other patient with Leo stent occlusion was managed conservatively due to his asymptomatic clinical status and presence of other comorbidities. There was no statistically significant difference in the involvement between Neuroform and Leo stents.

In a series of 14 patients with symptomatic intracranial stenosis, quantitative MRA (QMRA) showed low specificity and positive predictive value in detecting in-stent stenosis compared with our series. ${ }^{8}$ In this study, CE-MRA reformatted images showed better specificity and positive predictive value, though sensitivity and negative predictive value is low (-Table 2). As against QMRA, the CEMRA technique is easy to perform as well for interpretation, less subjected to artifacts from variable velocities. ${ }^{9}$ Our study show high sensitivity and specificity of CE-MRA source image for detection of significant delayed in-stent stenosis.

Limitations of the study were (1) small number of cases in the cohort, the findings needs to support by larger study, (2) the study was retrospective-prospective study. We suggest need for prospective blinded study. (3) CE-MRA showed 39\% false positivity for less than $50 \%$ stenosis. (4) The number of Solitaire AB (Covidien) and Enterprise stents (Codman) were very less, hence direct comparison between these two stents against other stents were not possible. (5) Larger field of view (including neck + brain) used for CE-MRA image acquisition likely result in spread of acquired data over larger pixel. This limitation could be overcome by using small FOV for intracranial circulation only. This modified technique may further decrease false positive cases of delayed in-stent stenosis, though this aspect was not studied in the current study.

\section{Conclusion}

Development of delayed in-stent stenosis in nonatherosclerotic intracranial stenting is $11 \%$. Even though CE-MRA source image analysis give false positive result in stenosis $<50 \%$, it is a good imaging tool for detection and follow-up of significant ( $>50 \%$ ) intracranial in-stent stenosis.

\section{Author Contributions}

Jayadevan Enakshy Rajan and Santhosh Kumar Kannath contributed in concept and design, data analysis, data interpretation, article preparation, and critical revision. Kamble Jayaprakash Harsha contributed in data analysis, data interpretation, article preparation, and critical revision.

\section{Conflict of Interest}

None.

\section{References}

1 SSYLVIA Study Investigators. Stenting of Symptomatic Atherosclerotic Lesions in the Vertebral or Intracranial Arteries (SSYLVIA): study results. Stroke 2004;35(06):1388-1392

2 Turk AS, Levy EI, Albuquerque FC, et al. Influence of patient age and stenosis location on wingspan in-stent restenosis. AJNR Am J Neuroradiol 2008;29(01):23-27

3 Yoon KW, Kim YJ. In-stent stenosis of stent assisted endovascular treatment on intracranial complex aneurysms. J Korean Neurosurg Soc 2010;48(06):485-489

4 Fiorella D, Albuquerque FC, Woo H, Rasmussen PA, Masaryk TJ, McDougall CG. Neuroform in-stent stenosis: incidence, natural history, and treatment strategies. Neurosurgery 2006;59(01): 34-42

5 Choo KS, Lee TH, Choi CH, Park KP, Kim CW, Kim S. Assessment of the intracranial stents patency and re-stenosis by 16 -slice CT angiography with optimized sharp kernel : preliminary study. J Korean Neurosurg Soc 2009;45(05):284-288

6 Hansberg T, Wong KSL, Droste DW, Ringelstein EB, Kay R. Effects of the ultrasound contrast-enhancing agent Levovist on the detection of intracranial arteries and stenoses in chinese by transcranial Doppler ultrasound. Cerebrovasc Dis 2002;14(02): 105-108

7 Vicenzini E, Puccinelli F, Ricciardi MC, Guidetti G, Delfini R, Lenzi GL. Contrast-enhanced transcranial color-coded duplex sonography versus computed tomography and magnetic resonance angiography in the follow-up of basilar stenting. J Ultrasound Med 2007;26(04):543-546

8 Thamburaj K, Cockroft K, Agarwal AK, Sabat S, Kalapos P. A comparison of magnetic resonance angiography techniques for the evaluation of intracranial aneurysms treated with stentassisted coil embolization. Cureus 2016;8(12):e909

9 Prabhakaran S, Warrior L, Wells KR, Jhaveri MD, Chen M, Lopes DK. The utility of quantitative magnetic resonance angiography in the assessment of intracranial in-stent stenosis. Stroke 2009; 40(03):991-993 